Session XXXX

\title{
Vertical Laboratories: Within Biomedical Engineering Courses and Across the Curriculum
}

\author{
Samantha J. Richerson, Daniel P. Cavanagh \\ Biomedical Engineering Program \& Department of Electrical Engineering / \\ Biomedical Engineering Program \& Department of Chemical Engineering \\ Bucknell University, Lewisburg, PA
}

Purpose:

In an effort to enable our students to further build their knowledge base experimentally, a new vertical laboratory method is currently being developed and implemented into the Introduction to Biomedical Engineering class at Bucknell University. This vertical laboratory centers on a few experimental setups that can be utilized to offer a range of hands-on experiences that vary in complexity and allow our student to focus on the concept being presented instead of the experimental set-up.

Introduction:

As in most introductory classes, many assumptions are made to simplify the concepts and problems encountered by students. As the class progresses, the assumptions are relaxed leading to more difficult concepts and problems. This process relies on the building of knowledge from a strong base and the adding of additional knowledge to supplement that base.

However, most laboratory protocols in the introductory classes are demonstration based. Students work on a protocol designed by the instructor that proves a concept learned in lecture. Each laboratory is frequently an island unto itself, with no building of conceptual knowledge from one lab to the next. Additionally, most of these laboratories have a detailed protocol, not allowing the student to design or experimentally learn concepts on their own.

In these laboratory experiences, basic skills into scientific inquiry or the scientific method are often overshadowed by procedural problems. Students spend much of their laboratory time on the nuances of equipment and the prescribed data collection instead of on the observation, prediction, and inference that is the basis of the scientific method. It has been shown that these skills of observation, measurement, prediction, and inference are those skills necessary to build a foundation for learning more complicated and integrated skills such as data analysis, interpretation, design of experiments and model formation ${ }^{1-3}$. These higher level skills are often a 
goal of curricula, yet the basis of knowledge needed to acquire these skills is not being met using current laboratory structures.

Historically there have been two types of laboratory experiences. Under the first, students are given a "plan of attack", where a set of prescribed experiments are given, data collection is required, and results are compared to the theoretical standard to determine how accurate or precise students were with respect to the "right" or theoretical answer. The second type of laboratory experience is the design. Students are given a design goal or objectives, and little or no supporting information on how to design. This is almost equivalent to dropping students in the deep end of the pool without teaching them how to swim.

There is currently a large gap between these two types of laboratory experiences. In the first experience, students are focused on how to get the equipment to give them the answer that most closely resembles the lecture material or the book. They are not taught how to design the experiment or analyze data in a way that gives information about the problem or concept that is being studied. The second method gives students no support such that they tend to flounder with the problem. Over a long period of trial and error they may succeed, however this often takes more than the prescribed 2 or 3 hour laboratory session. So how do we design laboratories that bridge this gap?

It is our contention that a set of laboratory experiments can be designed in a vertical manner to not only allow students to build conceptual knowledge from one laboratory to the next, but additionally, those experiments can increase in difficulty from a prescribed protocol, to one in which a complete design is required, even in an introductory class.

We have implemented this vertical lab structure in the Introduction to Biomedical Engineering class at Bucknell University during the fall semester of 2004. The class consisted of 13 sophomore BME majors. The class has an integrated lecture/laboratory format which meets five hours a week in two two-hour blocks, and a one hour lecture. Laboratories are generally carried out within the two hour block. The purpose of the course was to give students an introduction to some of the fundamental aspects and concepts of biomedical engineering including biofluid mechanics, biomedical mass transport, and bioinstrumentation. The goal of the laboratory was to reinforce technical concepts from class and to prepare the students for the final project that integrated concepts from these three areas.

The final project description (as given to the students) is provided below to further provide the reader with insight in to goals of the course.

The overall goal of this project is for each group to proceed through the process of designing and making experimental measurements within a fluid flow system. After constructing the recirculating system used in prior experimental protocols, each group will design their own additional modifications which will individualize each group's project. The objective of modifying the experiments is to create a setup with will permit your group to quantify the effect of the changes to the system. Each group will be monitoring pressures within a specific section of the fluid flow system. Hence, this project will require the utilization of skills gained in this course in the areas of fluid flow, pressure measurement, instrumentation, and 
data/signal analysis. While one goal of this project is to acquire useful data which can be quantified to examine the effects of the experimental modifications, an equally important goal is the process by which the project is completed. Poor design process with excellent results do not indicate successful project completion. To aid in the process of design, a design log will be kept to help detail the steps of your group's design.

Course Laboratories:

To provide the necessary skills needed to complete this project, the following laboratories were performed in a manner that allowed students to re-use many of the equipment set-ups previously seen, and hence focus on the concepts presented.

Example of Vertical Laboratory Structure:

Lab 1: Introduction to Hydrostatics

- Purpose: Design and construct an experimental setup to make a series of measurements to determine the conversion factor between two different units of pressure: psi and $\mathrm{cm} \mathrm{H}_{2} \mathrm{O}$. Also, the experimental relation between pressure and height will be examined. This is an open-ended exercise with no exact solution so groups need to work effectively as in a team setting in accomplishing the goals.

- Equipment and Methodology: Students use a pressure gauge, tubing, and fittings to determine the calibration curve between $\mathrm{cm}_{2} \mathrm{O}$ and psi. Topics such as repeatability and regression are also explored.

Lab 2: Pressure-Flow Relationships

- Purpose: Use a recirculating flow system to experimentally examine relationships between pressure, flow, viscosity, and geometry in pipe flow.

- Equipment and Methodology: Students use tubing, tubing connectors, 2 pressure gauges (from Lab 1), a fluid pump, rotameter, pulse dampener, and fluid reservoir to create a recirculating system. Students explore the dynamics of recirculating flow including the relationship between pressure drop and flow rate for varying tubing sizes and varying heights, calculations of Reynolds's number, and Poiseuille's equation. Additionally, effects of fittings on recirculating system were calculated.

Lab 3: Mass Balance Experiments

- Purpose: To apply conservation of mass principles to an experiment that models portions of the drug delivery process. The experiment models the constant infusion of a drug and allows monitoring of the concentration of the drug in the system. This experiment should yield data comparable to the theoretical model developed in class.

- Equipment and Methodology: Students use Vernier Data Acquisition System (to measure concentration), laptop PC connected to Vernier system, tubing, beakers, syringe pump, rotary pump, rotameter, pulse dampener (the underlined is from Lab 2), food coloring, and a stopwatch to create a recirculating system with an infusion of colored liquid. Concentration of the dye is measured at prescribed time intervals and students use that data to create a model and compare it to one developed in class. 
Lab 4:LabVIEW Introduction

- Purpose: To introduce our students to programming in LabVIEW.

- Equipment and Methodology: Students use computers and the LabVIEW 3 hour tutorial to learn the basics of programming in a graphical language including signal manipulation, sub-VI development, conditional loops, and data acquisition.

Lab 5: Sampling and Filtering Lab

- Purpose: To use Labview to acquire data and sample data. Data was acquired at various sampling rates to show the sampling theory. The data was also filtered using various low and high pass filters to see the effect of filtering and determine the cut-off frequency

- Equipment and Methodology: Students used their LabVIEW knowledge to build a vi that acquired, filtered, and saved data. A function generator supplied sine waves of varying frequency. As the students varied the sampling rate of the data acquisition, they were able to compare the actual frequency of the input waveform to the sampled frequency. Also, they were able to change the sampling types and determine the effect on waveforms of varying frequencies.

Lab 6: Introduction to Pressure Transducers

- Purpose: To acquire a voltage from a pressure transducer in a recirculating system, calibrate the voltage to a pressure measurement, filter and analyze the signal. The same recirculation system that was prepared in Lab 2 was re-utilized, the only change was the replacement of the pressure gages with pressure transducers.

- Equipment: All equipment in Lab 2. Additionally, computer with data acquisition capability, LabVIEW, DC power supply, and pressure transducers. Students applied the same calibration technique used in Lab 1 to calibrate the sensors. Then, the recirculating systems were built, and students measured the effect of flow rate on pressure drop. Signals were acquired using LabVIEW that the students wrote in lab 5. Signals were filtered using a low pass filter and analysis similar to Lab 2 was utilized. Additionally, students looked the frequency of the sinusoidal pressures produced by the rotatry pump and analyzed the relationship between frequency and flow rate.

Examples of Final Projects:

Students used a recirculating system with pressure transducers and LabVIEW programs similar to Laboratory 6 . They were required to calibrate their systems and use concepts from nearly all of the previous laboratories to predict the outcome of their experiments. Their predictions were to be compared to their data and if they did not match, probable reasons of error were to be discussed.

Example 1: Model of plaque buildup in arteries. This group created the reirculating system in laboratory 6. Calibration of the pressure transducers was undertaken using the same protocol as laboratory 1 . Two pressure transducers were placed between a length of tubing filled with a mesh of steel wool. They then introduced a solution of ferric ammonium citrate to the system. This chemical has a precipitate that this group believed would bind to the mesh and create a buildup of precipitate, mimicking the buildup of plaque in the body. Using LabVIEW, this group 
sampled data from three different concentrations of solute during 30 minute trials. They then correlated the change in pressure drop to the amount of buildup in the system and the concentration of the solute.

Example 2: Model of Abdominal Aortic Aneurysms (AAA). This group used different sized tubing to create a recirculating scaled model of the descending aorta. They used balloons to model the area of the aorta that was venerable to aneurysm. They then varied flow rate, position of the aneurysm (supraceliac region and infarenal region), and arterial clogging (as modeled by partial clamping of tubing), and measured the difference in pressure across the aneurysm. They used all of the data to create a model that predicts the occurrence of an AAA.

\section{Discussion:}

The purpose of vertical laboratories is to allow students to re-use equipment set-ups or concepts from previous laboratories in order to build on a foundation of knowledge. By using the same laboratory set-up, students become familiar with the equipment and are not focused on the proper use of the equipment during the laboratory period. Instead, they focus their attention of the concept or problem that the experiment teaches. Additionally, by re-presenting material/concepts/procedures over and over students retain more of that information for a longer period of time.

In the Introduction to Biomedical Engineering class that was presented here, a core experimental setup up has been designed which allows students to investigate a wide rage of fundamental biotransport concepts. While the students begin by investigating pressure-flow relationships in a recirculating system, they can then utilize the system to examine frictional losses and the mechanical energy balance. Finally, the students then incorporate solute transport into the system to examine unsteady mass balances and the associated dependence upon the fluid transport. Students are then introduced to instrumentation using LabVIEW and pressure transducers. These items are then incorporated into the same core experimental set-up to look at signals and filtering. All of these concepts are then reinforced in a design project that the students complete at the end of the semester. This vertical approach to the lab experiences hopefully serves to increase the students understanding of biotransport and bioinstrumentation fundamentals.

This vertical laboratory procedure was not only utilized within distinct section topics in class (biofluids), but it was used throughout the entire class, with the final projects as the culminating focus. Additionally, this methodology can be used throughout the curriculum. Experimental setups seen in one class can be used across the curriculum to further deeper conceptual knowledge. For example, the recirculating flow system seen in the Introduction to Biomedical Engineering course can be utilized in later Biotransport courses to teach higher level concepts.

Student Feedback

At the end of the semester project, feedback from the students was solicited. Below are some student comments:

- I really liked that there was no restriction on the topics of the projects. 
- I think we needed to move the pre-planning/design of the projects earlier in the semester so that we have more time for the experiment and data analysis.

- I liked the application of the labs into the projects.

- I like that we could order whatever parts/fittings we needed, but if we ordered them sooner, we would have had more time at the end for building/testing.

References:

1. Funk, H.J., Okey, J.R., Fiel, R.K., Jans, H.H., Sprague, C.S. (1979). Learning Science Process Skills. Duboque, IA: Kendall/Hunt.

2. Padilla, M.J. (1991). Science activities, process skills, and thinking. In S.M. Glynn, R.H. Yeany, and B.K. Britton (Eds.), The psychology of Learning Science. Lawrence Erlbaum Associates: Hillsdale, NJ.

3. Yap, K.C., and Yeany, R.H., (1988). Validation of hierarchial relationships among Piagetian Cognitive Modes and Integrated science process skills for difference cognitive reasoning levels. J Research in Science Teaching, 25, 247-281.

Biographical Information

SAMANTHA J. RICHERSON is an assistant professor of biomedical and electrical engineering at Bucknell University. Dr. Richerson teaches a range of biomedical and electrical engineering courses in addition to research that incorporates psychophysics into postural control testing in healthy and diabetic elders. She also researches theoretical models of neuronal signal transduction in the peripheral nervous system.

DANIEL P. CAVANAGH is an associate professor of biomedical and chemical engineering at Bucknell University in Lewisburg, PA. In addition to currently holding the Emmitt Memorial Chair in Biomedical Engineering, he is also Director of the Biomedical Engineering Program. Dr. Cavanagh teaches a variety of biomedical and chemical engineering courses in addition to researching intravascular gas embolism and dialysis. 